\title{
Effect of polymeric nanoparticles on dielectric and electro-optical properties of ferroelectric liquid crystals
}

\author{
A. Kumar, ${ }^{1,2}$ P. Silotia, ${ }^{2}$ and A. M. Biradar ${ }^{1}$ \\ ${ }^{1}$ Liquid Crystal Section, National Physical Laboratory, Dr. K. S. Krishnan Road, New Delhi 110012, India \\ ${ }^{2}$ Department of Physics and Astrophysics, University of Delhi, Delhi 110007, India
}

(Received 12 February 2010; accepted 16 May 2010; published online 21 July 2010)

\begin{abstract}
It has been observed that the polymeric nanoparticles, copolymer of polybenzene and anthracene (PBA NPs), can induce a homeotropic (HMT) alignment in various ferroelectric liquid crystal (FLC) mixtures. The HMT alignment of various FLCs is attributable to the fact that the anthracene molecules favor an upright orientation (with a little tilt) on the surfaces of indium tin oxide and this upright orientation of anthracene molecules works as a template to align FLC molecules homeotropically. It has been concluded that the addition of $\sim 0.5$ wt $\%$ of PBA NPs is enough to induce a HMT alignment in a FLC material Felix 17/100. The influence of the PBA NPs concentrations on the transition temperature, physical constants (such as spontaneous polarization, rotational viscosity, and response time) and dielectric relaxation processes of FLC material (Felix 17/100) has also been investigated. (C) 2010 American Institute of Physics. [doi:10.1063/1.3452355]
\end{abstract}

\section{INTRODUCTION}

The liquid crystal (LC) materials have proved themselves the most promising candidates in the field of highly growing electro-optical display as well as nondisplay devices. The homeotropically aligned LCs have been extensively used for LC displays (LCDs) such as large-area LCD televisions, information display devices, and digital displays in medical devices due to its unprecedented contrast ratio and wide viewing angle characteristics. ${ }^{1}$ The various techniques have been investigated to accomplish the homeotropic (HMT) alignment of LCs by the researchers around the world. ${ }^{2-8}$ It has been observed that the minute addition of nanomaterials (e.g., nanoparticles, nanotubes, etc.) to LC materials has improved many special characteristics in the form of frequency modulation response, ${ }^{9}$ nonvolatile memory effect, ${ }^{10}$ faster electro-optic response and low threshold switching, ${ }^{11,12}$ low driving voltage, ${ }^{13}$ enhanced photoluminescence, ${ }^{14}$ and reduced residual dc. ${ }^{15}$ The HMT alignment of LCs by incorporating NPs has emerged a fascinating field of research as it provides a better tool to achieve defect free alignment of LCs without use of any alignment technique. It has been observed that polyhedral oligomeric silsesquioxanes (POSS) nanoparticles can induce a HMT alignment of nematic LC with positive anisotropy. ${ }^{5}$ The effect of the POSS concentration on the electro-optical and dielectric properties of the LC has also been monitored. ${ }^{16}$ The authors have concluded that the doping of $\sim 1$ wt $\%$ of POSS NPs is enough to induce a uniform HMT alignment of the LC for best electro-optical properties.

Recently, we have observed that the NPs of copolymer of polybenzene and pentacene (PNPs) can induce a HMT alignment in various ferroelectric LC (FLC) mixtures. ${ }^{17} \mathrm{We}$ proposed that the HMT alignment of FLC mixtures is due to the interaction of pentacene molecules, which favors an upright orientation on indium tin oxide (ITO) substrate with FLC molecules. It has been found that the addition of PNPs into FLCs has also influenced the physical parameters and the $\mathrm{SmC}^{*}-\mathrm{SmA}^{*}$ phase transition temperature $\left(\mathrm{T}_{\mathrm{C}}\right)$ remarkably. To effectively employ the PNPs induced HMT alignment of FLCs, the characteristics of PNPs added FLCs must be analyzed in detail. To minimize the changes in physical constants and $\mathrm{T}_{\mathrm{C}}$ for PNPs doped FLCs, we synthesized the NPs of copolymer of polybenzene and anthracene (PBA NPs) by replacing the molecules of pentacene by anthracene in the synthesis process of PNPs. ${ }^{17}$ The idea behind this replacement was that both, pentacene and anthracene, belong to acenes and anthracene molecules are smaller in size. It is thought that the changes in the $\mathrm{T}_{\mathrm{C}}$ and physical constants of the FLC materials doped with PBA NPs would be least due to smaller size of anthracene molecules present in the PBA NPs. Anthracene $\left(\mathrm{C}_{14} \mathrm{H}_{10}\right)$ and its derivative are well known organic semiconductors that have been successfully used to fabricate blue organic light emitting diodes. ${ }^{18-20}$ The growth morphology of anthracene on various surfaces has extensively been investigated. Gregor et al. reviewed the growth of planar aromatic molecules with extended $\pi$-systems namely anthracene, tetracene, pentacene, etc. on solid substrates. ${ }^{21}$ They demonstrated the molecular orientation of pentacene thin films on $\mathrm{Ag} / \mathrm{Si}$ (111) by using scanning tunneling microscopy. They also showed that in the first monolayer, the molecules adopt planar adsorption geometry, further deposition is found to favor an upright orientation with the molecular axis being tilted away from the surface. The growth of thin films with a bulk like structure is favored, with the molecules adsorbed in such a fashion that it maximizes the intermolecular interactions. A consequence of this rule is that very often the long axis of molecule is found to be orientated normal to the substrate. The thin film growth of anthracene films on Si (111) surfaces has been studied by photoemission electron microscopy. ${ }^{22}$ It has been observed that initially, a layer of flat lying molecules chemisorbs on the surface, subsequent growth of fractal islands with standing up molecules proceeds on the top of this layer. The au- 
thors also suggested that the thin film growth of anthracene is similar to the growth of pentacene molecules on silicon.

In this paper, we have presented the quantitative analysis of PBA NPs induced HMT alignment of FLCs. It has been pursued by monitoring the influence of PBA NPs concentrations on the dielectric properties, transition temperature and physical constants of PBA NPs doped FLC mixture. The optimization for the PBA NPs concentrations to achieve good HMT alignment as well as electro-optical properties of FLCs has also been discussed.

\section{EXPERIMENTAL DETAIL}

The PBA NPs were synthesized by the same chemical method that was used to synthesize PNPs. ${ }^{17}$ The synthesized PBA NPs are soluble in many organic solvents like chlorobenzene, n-methyl-1-pyrollidine and chloroform, etc. The mixture of FLCs and PBA NPs has been prepared in different wt/wt ratios such as $0.1 \%, 0.5 \%, 1 \%, 2 \%, 5 \%$, and $10 \%$. The prepared mixture was then introduced into the LC sample cells by means of capillary action at temperatures just above the isotropic transition temperatures of the respective FLC materials. We used the sample cells consisting of highly conducting ( $\sim 30 \Omega / \square)$ ITO coated glass plates. The ITO patterns used as electrodes were prepared by photolithographic technique. The thickness between the two plates was maintained around $5 \mu \mathrm{m}$ using Mylar spacers. The LC materials were filled in isotropic phase and then cooled gradually to room temperature. The dielectric studies of the FLC materials have been performed using an impedance analyzer 6540 A (Wayne Kerr, U.K.). The phase sequences of FLC mixtures used are as follows:

$$
\begin{aligned}
& -8{ }^{\circ} \mathrm{C} \quad 15-18{ }^{\circ} \mathrm{C} \quad 75^{\circ} \mathrm{C} \quad 92-103{ }^{\circ} \mathrm{C} \\
& \text { cryt } \leftrightarrow \mathrm{SmC}^{*} \leftrightarrow \mathrm{SmA}^{*} \leftrightarrow \mathrm{N} \leftrightarrow \text { iso (Felix 20), } \\
& -28^{\circ} \mathrm{C} \quad 73^{\circ} \mathrm{C} \quad 77^{\circ} \mathrm{C} \quad 84-87^{\circ} \mathrm{C} \\
& \text { cryt } \leftrightarrow \mathrm{SmC}^{*} \leftrightarrow \mathrm{SmA}^{*} \leftrightarrow \mathrm{N} \leftrightarrow \quad \text { iso (Felix 17/100), } \\
& -21{ }^{\circ} \mathrm{C} \quad 56{ }^{\circ} \mathrm{C} \quad 67^{\circ} \mathrm{C} \quad 73^{\circ} \mathrm{C} \\
& \text { cryt } \leftrightarrow \mathrm{SmC}^{*} \leftrightarrow \mathrm{SmA}^{*} \leftrightarrow \mathrm{N} \leftrightarrow \text { iso (CS 1016), } \\
& -7{ }^{\circ} \mathrm{C} \quad 64{ }^{\circ} \mathrm{C} \quad 82{ }^{\circ} \mathrm{C} \quad 91^{\circ} \mathrm{C} \\
& \text { cryt } \leftrightarrow \mathrm{SmC}^{*} \leftrightarrow \mathrm{SmA}^{*} \leftrightarrow \mathrm{N} \leftrightarrow \text { iso (CS 1026), } \\
& 61{ }^{\circ} \mathrm{C} \quad 91{ }^{\circ} \mathrm{C} \quad 115^{\circ} \mathrm{C} \\
& \text { cryt } \leftrightarrow \mathrm{SmC}^{*} \leftrightarrow \mathrm{SmA}^{*} \leftrightarrow \mathrm{N} \leftrightarrow \text { iso }(\mathrm{SCE}-9) .
\end{aligned}
$$

The determination of spontaneous polarization $\left(\mathrm{P}_{\mathrm{S}}\right)$, rotational viscosity $(\eta)$, and electro-optical response time $\left(\tau_{\mathrm{R}}\right)$ has been performed using an automatic LC tester (ALCT, Instec, USA). For optical micrographs, the sample was mounted on a polarizing microscope (Ax-40, Carl Zeiss Germany) and the transmission of normally incident polarized light through the sample was observed.

\section{RESULTS AND DISCUSSIONS}

We have studied the PBA NPs induced HMT alignment of different FLC mixtures using various experimental techniques. In case of HMT alignment, the LC molecules attain an orientation perpendicular to the substrate surface and yield completely dark field of view under crossed polarizers.

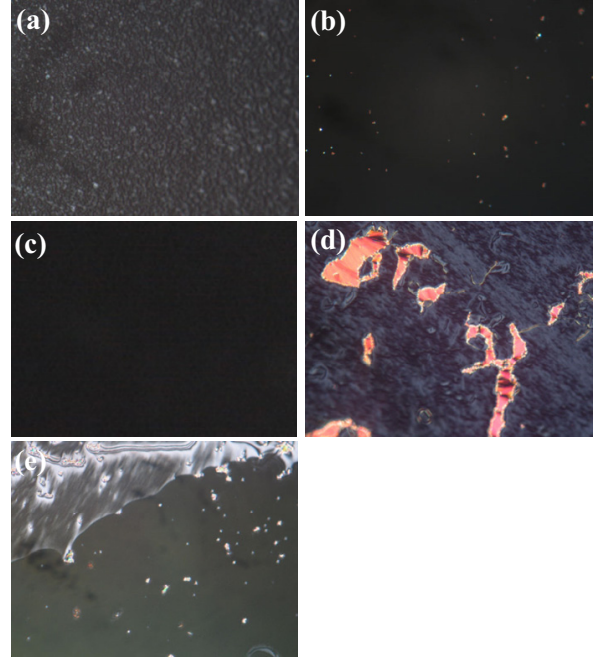

FIG. 1. (Color online) Optical micrograph of (a) Felix 17/100, (b) Felix-20, (c) FLC CS 1016, (d) FLC CS 1026, and (e) FLC SCE 9 doped with $\sim 0.5$ wt $\%$ of PBA NPs at room temperature.

It has been observed earlier that a FLC cell having pitch value less than cell thickness, the macroscopic birefringence disappear between the crossed polarizers except for a slight optical rotatory power. Hence, the light transmission is almost zero and one observe completely dark field of view under crossed polarizers. ${ }^{23}$ The optical micrographs recorded using polarizing microscope of different FLC materials doped with $\sim 0.5$ wt $\%$ of PBA NPs has been shown in Fig. 1. One can clearly see from the figure that for all the FLC mixtures studied, PBA NPs have induced a HMT alignment. However, in PBA NPs assisted HMT alignment, the long molecular axis of FLC molecules is not exactly perpendicular to the substrate surfaces but have some tilted orientation. Figure 1 also clarifies that the HMT alignment induced by PBA NPs is similar to that induced by PNPs observed earlier by us. ${ }^{17}$ It is worth to notice here that this PBA NPs induced HMT alignment of FLCs has been observed only in the regions where ITO was present, whereas in non-ITO regions, the homogeneous (HMG) alignment is favored. Figure 2 shows the optical micrographs of Felix 17/100 doped with different concentrations of PBA NPs. In the absence of an alignment layer, the FLC molecules align in a random fashion resulting in a bright multidomain texture for pure FLC cell as can be seen from Fig. 2(a). The induced alignment capability has been enhanced on increasing the PBA NPs concentration. The more and more FLC molecules tend to align homeotropically as the concentrations of PBA NPs is increased. The different alignments induced with different PBA NPs concentrations has been shown in Figs. 2(b)-2(g). A hybrid alignment showing the presence of both, HMG and HMT aligned domains has been observed under the crossed polarizers for the Felix $17 / 100$ added with 0.1 wt $\%$ of PBA NPs. A uniform HMT alignment of Felix 17/100 material has been observed in the case of 0.5 wt $\%$ PBA NPs doped Felix $17 / 100$ material as can be seen from Fig. 2(c). As the concentration of PBA NPs increased above 0.5 wt $\%$, the excess nanoparticles aggregated to form clusters as shown in Figs. 2(d)-2(g). The HMT alignment of Felix 17/100 material corresponding to different $\mathrm{PBA}$ NPs concentrations has also 


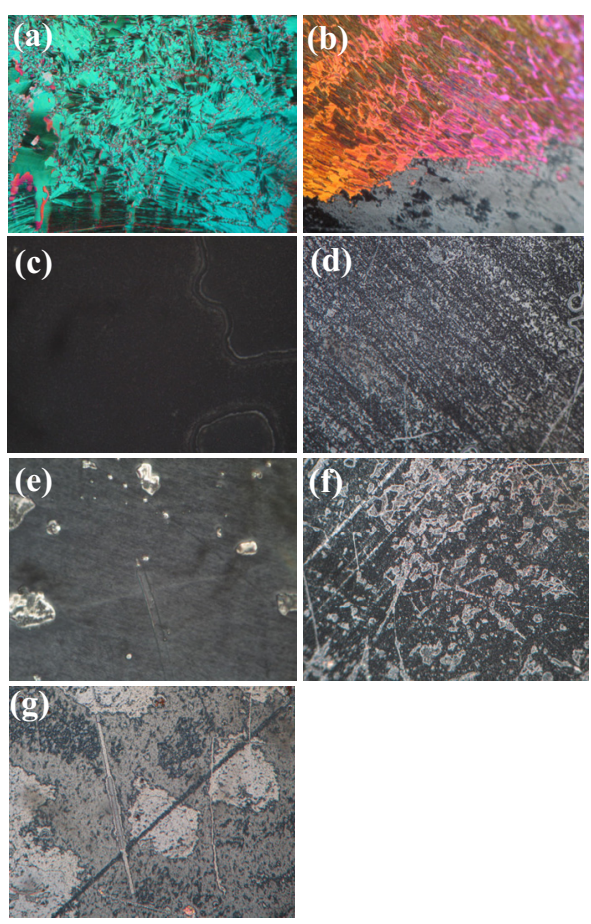

FIG. 2. (Color online) Optical micrographs of Felix 17/100 cells doped with (a) 0 wt $\%$, (b) $0.1 \mathrm{wt} \%$, (c) $0.5 \mathrm{wt} \%$, (d) 1 wt \%, (e) 2 wt $\%$, (f) 5 wt $\%$, and (g) $10 \mathrm{wt} \%$ of PBA NPs at room temperature. been confirmed by dielectric relaxation spectroscopy. The behavior of dielectric permittivity $\left(\varepsilon^{\prime}\right)$ with frequency at room temperature $\left(25^{\circ} \mathrm{C}\right)$ by applying different dc biases for Felix 17/100 material doped with different PBA NPs concentrations has been shown in Figs. 3(a)-3(f). The insets of these figures show the corresponding behavior of dielectric loss factor $(\tan \delta)$. It is clearly reflected from Fig. 3 that the value of $\varepsilon^{\prime}$ does not get suppressed even at higher values of applied voltages suggesting the alignment to be HMT for PBA NPs concentrations 0.5 wt $\%$ and above. From Fig. $3(\mathrm{a})$, it is clear that the value of $\varepsilon^{\prime}$ is larger for $0.1 \mathrm{wt} \%$ PBA NPs doped Felix 17/100 and it decreases on increasing the dc biases which suggest that $0.1 \mathrm{wt} \%$ of PBA NPs is not enough to induce HMT orientation of all the FLC molecules. The larger value of $\varepsilon^{\prime}$ has been observed due to the presence of some HMG aligned domains of FLC molecules. In the HMG alignment, the FLC molecules align parallel to the substrate surface giving larger values of $\varepsilon^{\prime}$ due to the contribution of Goldstone mode (GM) which can be suppressed by applying dc bias across the FLC cell. The FLC molecules align normal to the substrate surface in HMT aligned cells resulting in the lower value of $\varepsilon^{\prime}$ due to molecular rotations around their short molecular axis. The dielectric relaxations of this molecular mode appear in the $10^{4}-10^{6} \mathrm{~Hz}$ frequency range which can be seen from inset of Figs. 3(b)-3(f). How-
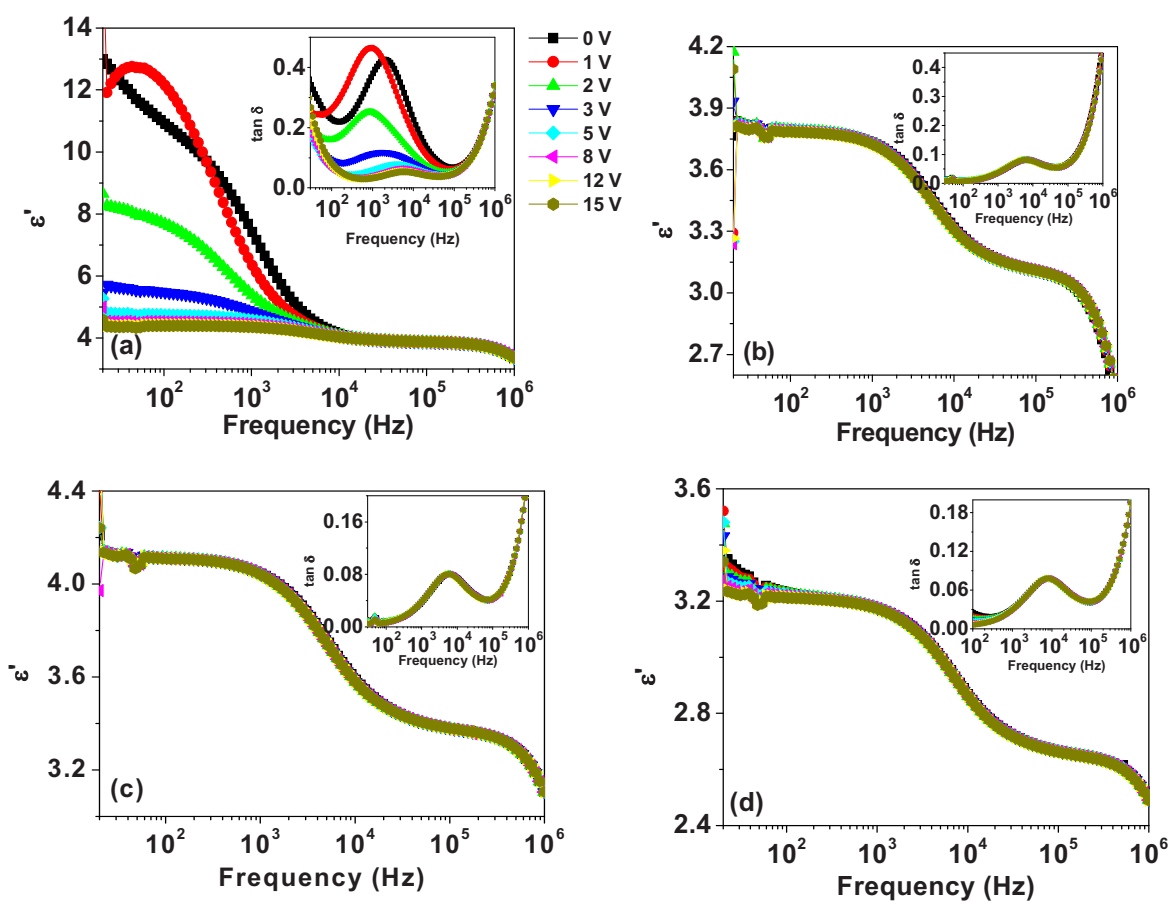

FIG. 3. (Color online) Behavior of the dielectric permittivity $\left(\varepsilon^{\prime}\right)$ and dielectric loss factor $(\tan \delta$ ) (shown in the inset) of Felix 17/100 doped with (a) 0.1 wt $\%$, (b) 0.5 wt $\%$, (c) 1 wt $\%$, (d) 2 wt \%, (e) $5 \mathrm{wt} \%$, and (f) 10 wt \% with applied voltage at room temperature. The value of applied bias is same in all figures (and inset) as in case of Fig. 3(a).
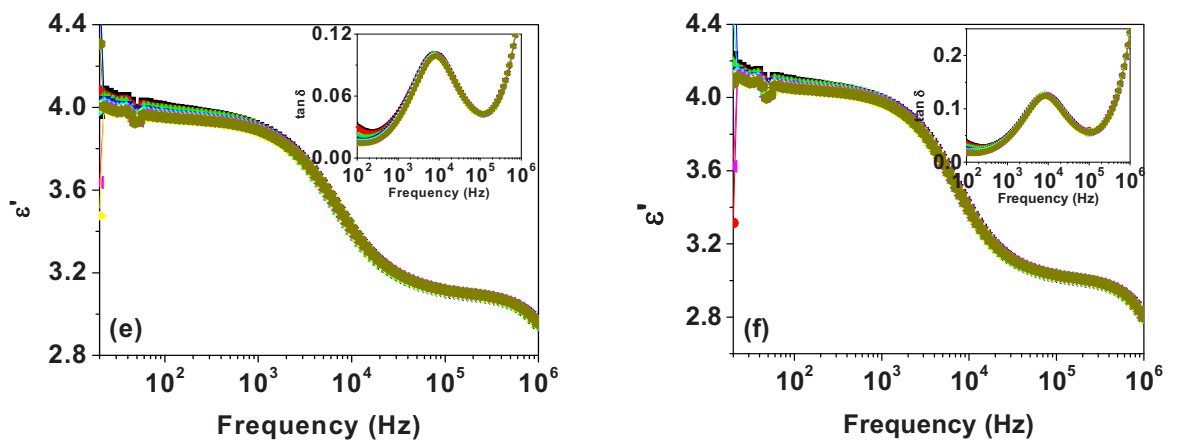

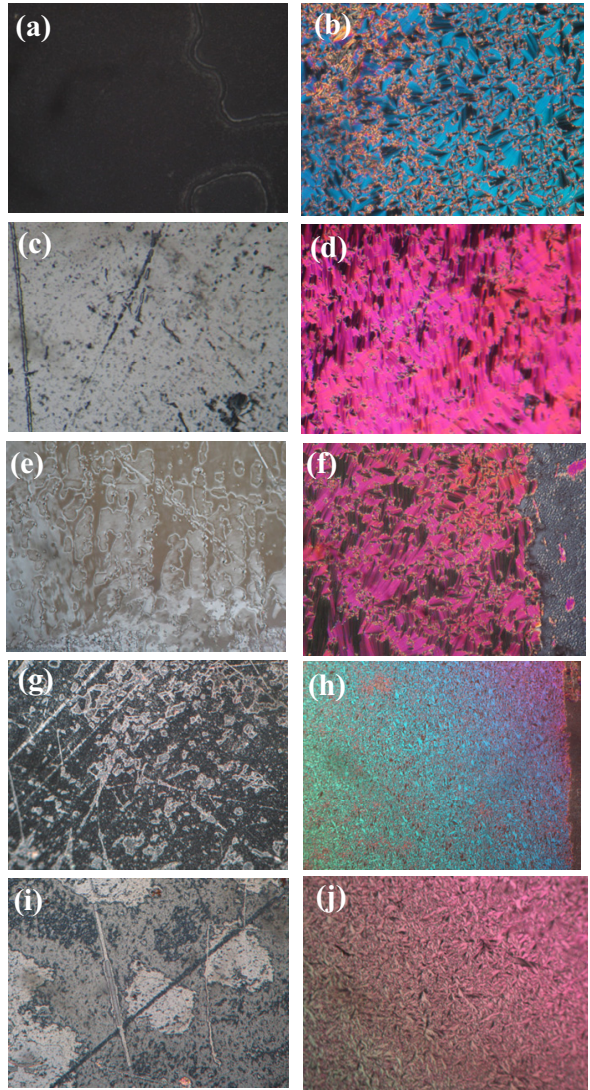

FIG. 4. (Color online) Polarizing optical micrographs showing the transformation from HMT to HMG configuration of Felix 17/100 doped with different PBA NPs concentrations: (a) $0.5 \mathrm{wt} \%$ at $0 \mathrm{~V}$, (b) $0.5 \mathrm{wt} \%$ at $38 \mathrm{~V}$, (c) 0.1 wt $\%$ at $0 \mathrm{~V}$, (d) $0.1 \mathrm{wt} \%$ at $40 \mathrm{~V}$, (e) 2 wt $\%$ at $0 \mathrm{~V}$, (f) 2 wt $\%$ at $42 \mathrm{~V}$, (g) 5 wt $\%$ at $0 \mathrm{~V}$, (h) $5 \mathrm{wt} \%$ at $45 \mathrm{~V}$, (i) 10 wt $\%$ at $0 \mathrm{~V}$, and (j) 10 wt $\%$ at $53 \mathrm{~V}$.

ever, in case of 0.1 wt \% PBA NPs doped Felix 17/100, the alignment is not completely HMT and hence GM has been appeared due to the presence of HMG aligned domains of FLC molecules [inset of Fig. 3(a)]. In HMT alignment, the stretching or rotation of the molecules is less along short molecular axis. So, the molecule does not experience the effect of bias in this alignment configuration. And so, on the application of bias, the $\varepsilon^{\prime}$ value does not get suppressed for the HMT aligned LC cells because molecules are standing on substrates. These effects could be more visualized in dielectric measurements with HMT alignment by using some other technique, e.g., alignment achieved by the application of magnetic field. ${ }^{24}$

It is noticeable that the HMT aligned FLC cells can be transformed into HMG configuration by applying certain amount of electric field across the cell electrodes. The transformation from HMT to HMG configuration has been favored in LC materials having negative dielectric anisotropy $(\Delta \varepsilon)$. On the application of sufficient electric field across the cell, the FLC molecules attain orientations parallel to the substrate surface due to coupling of the dipole moment along short axis with the applied electric field. Figure 4 shows the optical micrographs indicating the transformation from HMT to HMG configurations for Felix 17/100 doped with different concentrations of PBA NPs. It has been observed that the value of dc bias field needed to transform the FLC cell from
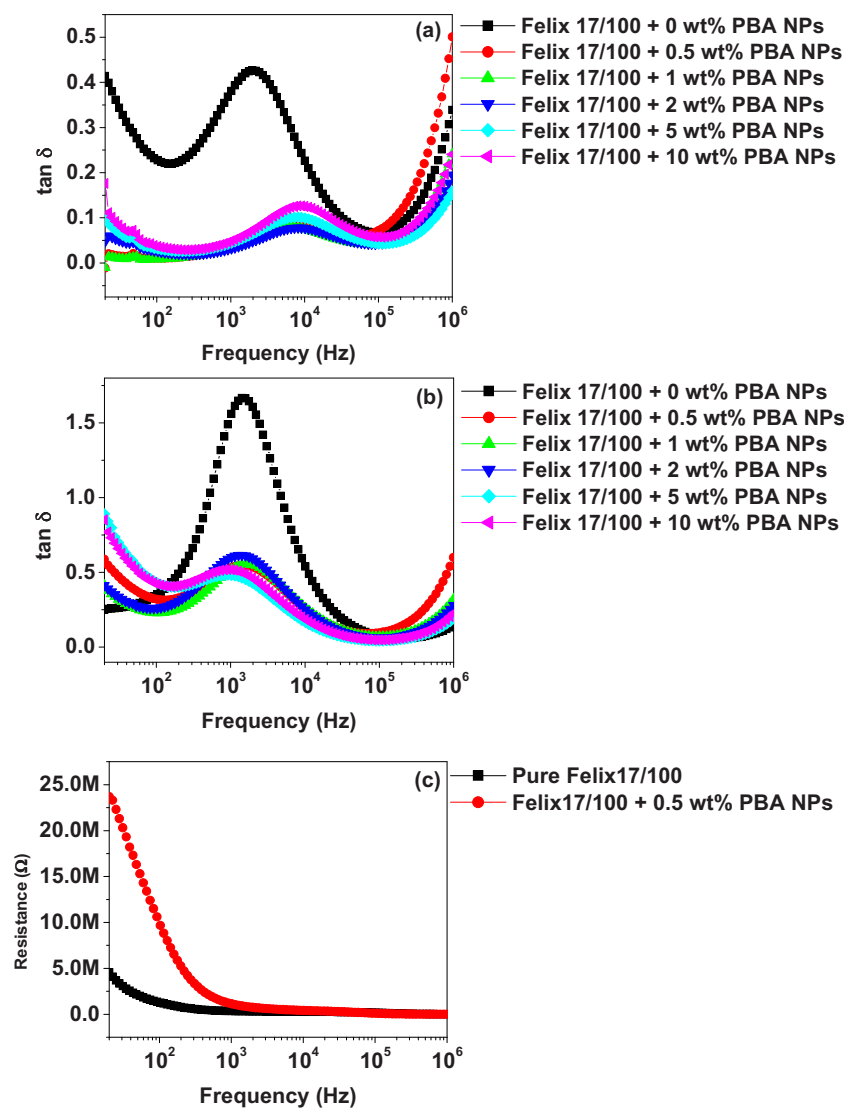

FIG. 5. (Color online) Comparison of dielectric loss factor ( $\tan \delta$ ) for different concentrations of PBA NPs doped Felix 17/100 in (a) HMT configuration, (b) HMG configuration, and (c) comparison of resistance of pure and 0.5 wt \% PBA NPs doped Felix 17/100 at room temperature.

HMT to HMG configuration depends on the concentrations of PBA NPs added to the Felix 17/100 material. The number of FLC molecules per unit volume of the sample cells goes on decreasing and hence the dipole moment per unit volume decreases on increasing the PBA NPs concentrations into the Felix 17/100 material. Hence, the value of dc bias needed for the transformation has been increased with the concentration of PBA NPs. Also, on increasing the concentration of PBA NPs, the molecular interaction between PBA NPs and FLC molecules increases and hence the more bias is required to transform the FLC molecules from HMT to HMG configuration. This electric field induced transformation of the FLC cells has been found irreversible to the field application, i.e., it does not come back to HMT configuration on removal of the transforming field. The FLC cells transformed in HMG configuration can again be transformed into HMT alignment only by heating the cell to isotropic temperature and cooling slowly. This again confirms the favorable upright orientation of PBA NPs on ITO surface. This transformation can be used to determine the dielectric anisotropy of the FLC materials very accurately using the same sample cell. ${ }^{17}$

The effect of different PBA NPs concentrations on $\tan \delta$ of the Felix 17/100 material in both (HMT and HMG) the configurations have been observed at room temperature. Figures 5(a) and 5(b) show the variation in $\tan \delta$ with frequency in HMT and HMG configurations, respectively, for Felix 17/ 100 doped with different concentrations of PBA NPs. It has 

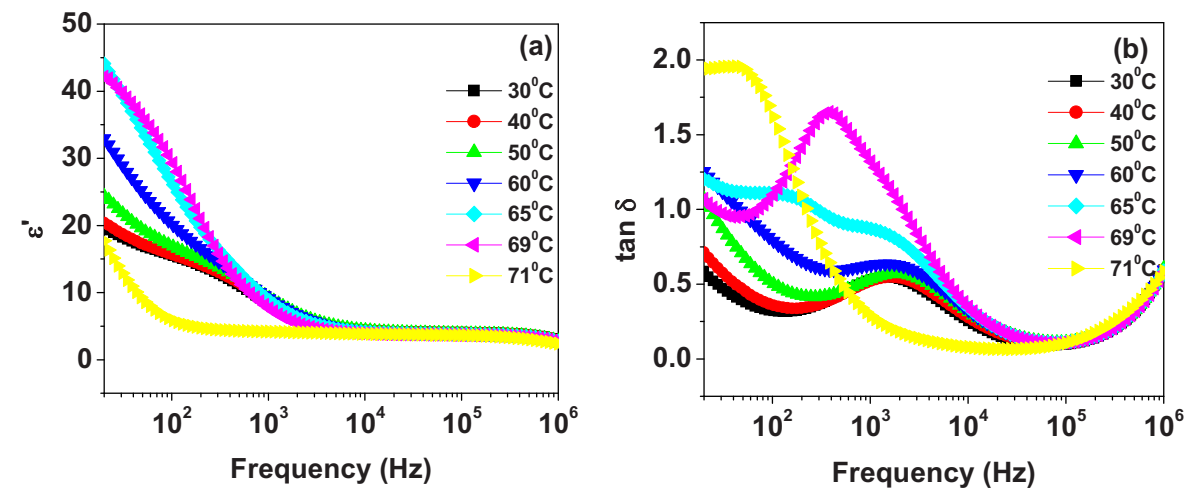

FIG. 6. (Color online) Behavior of (a) dielectric permittivity $\left(\varepsilon^{\prime}\right)$ and (b) dielectric loss factor $(\tan \delta)$ of 0.5 wt $\%$ PBA NPs doped Felix 17/100 with temperature in HMG configuration.

been reflected from Figs. 5(a) and 5(b) that the value of $\tan \delta$ in case of PBA NPs added Felix 17/100 has been reduced remarkably in both HMT and HMG configurations. The reduction in $\tan \delta$ might be due to the trapping of ions by PBA NPs and hence reducing the conductivity effects. The reduction in the conductivity has been reflected from Fig. 5(c) which shows the variation in resistance with frequency for pure and PBA NPs doped Felix 17/100 at room temperature. It is clear from the Fig. 5(c) that the resistance has been increased remarkably in the case of PBA NPs added Felix 17/100. We also studied the effect of PBA NPs concentrations on dielectric relaxation processes of Felix 17/100 with temperature. Figure 6 shows the behavior of $\varepsilon^{\prime}$ and tan $\delta$ for Felix $17 / 100$ material doped with 0.5 wt $\%$ of PBA NPs with temperature. It is clear from Fig. 6(a) that the $\varepsilon^{\prime}$ increases on increasing the temperature and reaches to higher values as $T_{C}$ is approached. It has been found that the dielectric strength attains higher values near $\mathrm{T}_{\mathrm{C}}$ whereas, in most of the conventional FLCs, it decreases with increasing the temperature in $\mathrm{SmC}^{*}$ phase and falls rapidly at $\mathrm{T}_{\mathrm{C}}$. This behavior of dielectric strength might be due to dominating ionic conductance in the case of PBA NPs doped Felix 17/ 100 which is increased on increasing the temperature. Another observance in support to this ionic effect has also been come into the picture. The dielectric strength has been decreased at $\mathrm{T}_{\mathrm{C}}$ and it increases rapidly as temperature is increased in $\mathrm{SmA}^{*}$ phase. To analyze the effect of ionic conductance, the variation in $\tan \delta$ with frequency at different temperature has been obtained [Fig. 6(b)]. The increase in the dielectric strength in $\mathrm{SmA}^{*}$ phase is due to the ionic conductance as the low frequency relaxation modes can easily be seen from Fig. 6(b). This ionic effect has been enhanced due to the doping of PBA NPs into the Felix 17/100 and it increases on increasing the doping concentrations of PBA NPs into the Felix 17/100 material. Figure 7 shows the behavior of $\varepsilon^{\prime}$ and $\tan \delta$ for Felix 17/100 material doped with $10 \mathrm{wt} \%$ of PBA NPs with temperature. It is reflected from Fig. 7(b) that the value of $\tan \delta$ has been increased much in $\mathrm{SmA}^{*}$ phase, along with a small increment of the same in $\mathrm{SmC}^{*}$ phase. While taking the measurements of $\tan \delta$ for PBA NPs doped Felix 17/100 material, a low frequency mode (say mode $\mathrm{X}$ ) in $\mathrm{SmC}^{*}$ has been appeared in all the Felix 17/100 cells doped with different concentrations of PBA NPs. It has been found that the relaxation frequency $\left(\nu_{\mathrm{R}}\right)$ of mode $\mathrm{A}$ increases with increasing the temperature and get merged with $\mathrm{GM}$ at $\mathrm{T}_{\mathrm{C}}$. This new mode $\mathrm{X}$ has been appeared in $\mathrm{SmC}^{*}$ phase and the values $\nu_{\mathrm{R}}$ of mode $\mathrm{X}$ has been found to be very near to GM. It was thought that the mode $\mathrm{X}$ might have appeared due to ionic conductance because of the addition of PBA NPs into the Felix 17/100 material. To verify this, we made an unaligned sample (cell without making any surface treatment) of pure Felix 17/100 material and taken the measurement of $\tan \delta$. Figure 8 shows the behavior of $\varepsilon^{\prime}$ and $\tan \delta$ for unaligned cell of pure Felix $17 / 100$ material with temperature. The mode $X$ has also been appeared much prominently in the case of pure Felix 17/100 material as can be seen from Figs. 8(a) and 8(b). The unusual dips in the $\varepsilon^{\prime}$ can be seen clearly [Fig. 8(a)] indicating the occurrence of mode $\mathrm{X}$ along with the $\mathrm{GM}$ in $\mathrm{SmC}^{*}$ phase of the pure Felix 17/100 material. The $\nu_{R}$ of mode $X$ has been found to increase with increasing the temperature as can be seen from Fig. 8(b). Dwivedi et al. studied the dielectric properties of an anti-FLC material (4B6Bi) in $\mathrm{SmC}_{\mathrm{A}}^{*}, \mathrm{SmC}^{*}$ and $\mathrm{SmA}^{*}$ phase. ${ }^{25}$ They observed one low frequency $(\sim 10 \mathrm{~Hz})$ absorption peak along with the $\mathrm{GM}$ in $\mathrm{SmC}^{*}$
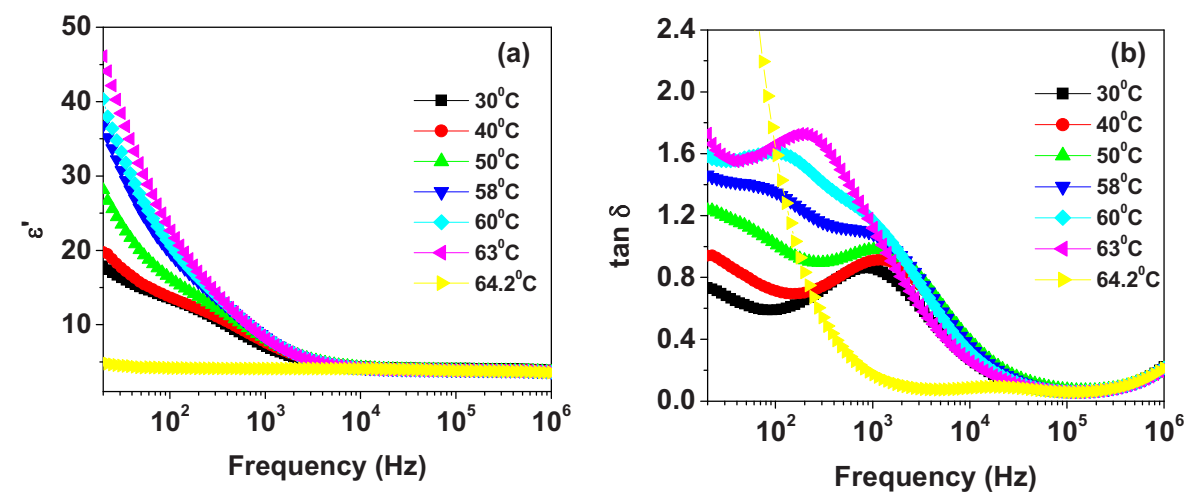

FIG. 7. (Color online) Behavior of (a) dielectric permittivity $\left(\varepsilon^{\prime}\right)$ and (b) dielectric loss factor $(\tan \delta)$ of $10 \mathrm{wt} \%$ PBA NPs doped Felix 17/100 with temperature in HMG configuration. 


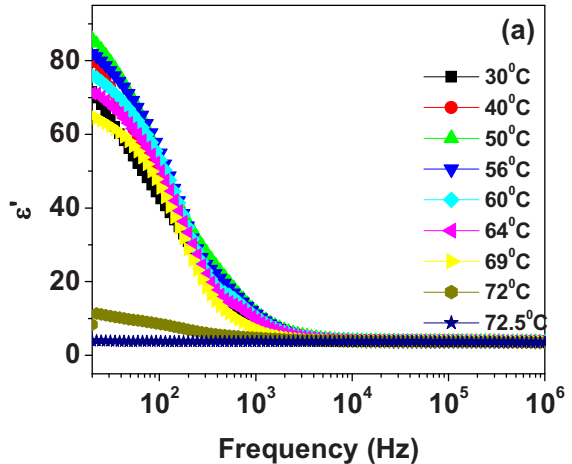

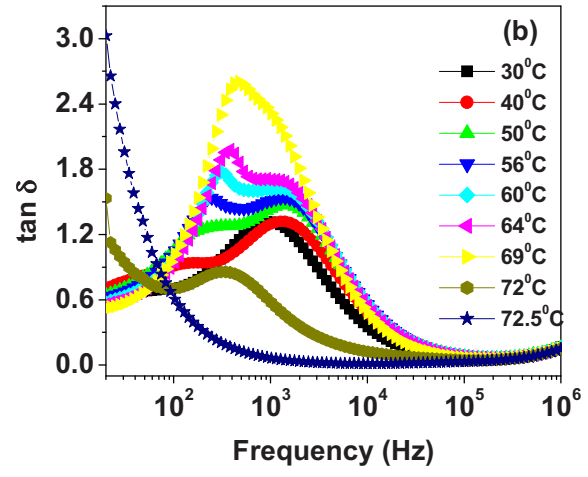

FIG. 8. (Color online) Behavior of (a) dielectric permittivity $\left(\varepsilon^{\prime}\right)$ and (b) dielectric loss factor $(\tan \delta)$ of unaligned Felix 17/100 sample cell with temperature in HMG configuration. phase of the material. They demonstrated that low frequency absorption peak was not due to the ionic conductance as it has not been observed in $\mathrm{SmA}^{*}$ phase where it is expected. In the case of pure Felix 17/100 material, the mode $X$ could not be assigned a relaxation mode due to ionic conductance as mode $\mathrm{X}$ has not been observed in $\mathrm{SmA}^{*}$ phase. However, in the case of lower concentrations of PBA NPs (below 0.5 wt $\%$ ) added Felix 17/100, a low frequency mode has been observed in $\mathrm{SmA}^{*}$ phase in the frequency regime in which it was appeared in $\mathrm{SmC}^{*}$ phase. The relaxation frequency of this mode increases on increasing the temperature which is the characteristics of the relaxations due to ionic conductance. $^{26}$ The occurrence of the low frequency mode is dominant for lower concentrations of PBA NPs added Felix $17 / 100$ material whereas it has been suppressed for higher
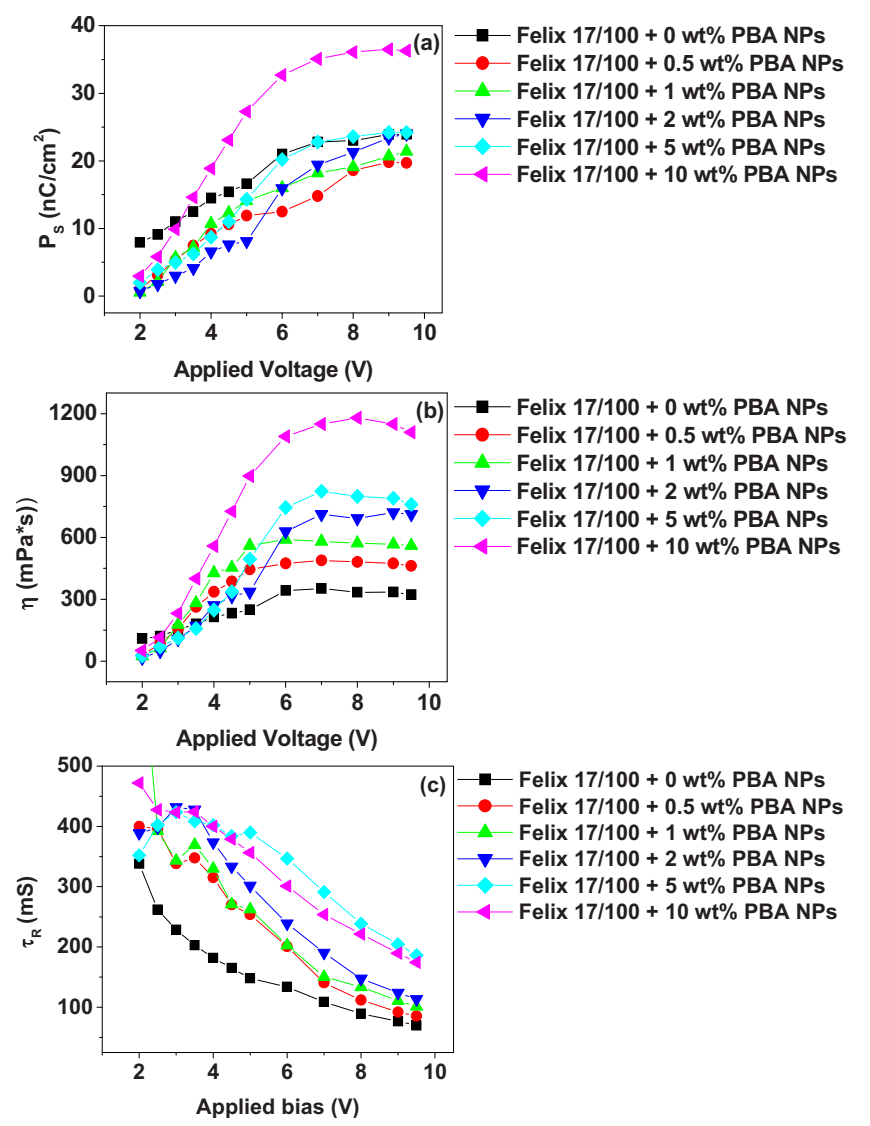

FIG. 9. (Color online) Behavior of (a) spontaneous polarization $\left(\mathrm{P}_{\mathrm{S}}\right)$, (b) rotational viscosity $(\eta)$, and $(\mathrm{c})$ response time $\left(\tau_{\mathrm{R}}\right)$ of Felix $17 / 100$ doped with different concentrations of PBA NPs with applied voltage. concentrations of PBA NPs. However, the observance of low frequency mode (mode $\mathrm{X}$ ) in $\mathrm{SmC}^{*}$ phase of various FLC mixtures needs serious investigations and the work is in progress which would be published elsewhere.

The influence of the concentrations of PBA NPs on the physical parameters of the Felix 17/100 has also been investigated. To observe the effects of different PBA NPs concentrations on physical parameters of the Felix 17/100, we determined the values of the physical constants for pure and PBA NPs doped Felix 17/100. Figure 9(a) shows the variation in $\mathrm{P}_{\mathrm{S}}$ of pure and different concentrations of PBA NPs doped Felix 17/100 after transforming them from HMT to HMG configurations. It is clear from the Fig. 9(a) that the value of $\mathrm{P}_{\mathrm{S}}$ has been decreased for 0.5 wt $\%$ PBA NPs and then starts increasing as the concentration of PBA NPs increases above $0.5 \mathrm{wt} \%$. However, the value of $\mathrm{P}_{\mathrm{S}}$ for PBA NPs concentrations below $10 \mathrm{wt} \%$ is less than that for pure Felix $17 / 100$ which can be due to the fact that by increasing the concentration of PBA NPs, the induced dipole moment of PBA NPs increases the $P_{S}$ value. Figure 9(b) shows the variation in $\eta$ with applied biases for Felix 17/100 material doped with different concentrations of PBA NPs. It is clear from the figure that the $\eta$ increases as the concentration of PBA NPs are increased. The increase in $\eta$ is least for the PBA NPs concentration of $0.5 \mathrm{wt} \%$. The value of $\tau_{\mathrm{R}}$ of Felix 17/100 doped with different concentrations of PBA NPs has also been studied. It is clear from the Fig. 9(c) that the $\tau_{\mathrm{R}}$ increases with increasing PBA NPs concentrations. The increase in the $\tau_{\mathrm{R}}$ on increasing the PBA NPs concentrations is the combined effect of decrease in $\mathrm{P}_{\mathrm{S}}$ and increase in $\eta$ values. The effect of PBA NPs concentrations on the $\mathrm{SmC}^{*}-\mathrm{SmA}^{*}$ transition temperature $\left(\mathrm{T}_{\mathrm{C}}\right)$ has also been observed by dielectric relaxation spectroscopy. The value of $\mathrm{T}_{\mathrm{C}}$ has been lowered on increasing the concentrations of PBA NPs as can be seen from Fig. 10. However, the lowering in the $\mathrm{T}_{\mathrm{C}}$ has been found to be small in comparison to the change due to the doping of PNPs (Refs. 17) and the lowering of $\mathrm{T}_{\mathrm{C}}$ is minimum $\left(\sim 3{ }^{\circ} \mathrm{C}\right)$ for $0.5 \mathrm{wt} \%$ doping concentration of PBA NPs. On the basis of the changes in the physical parameters and $\mathrm{T}_{\mathrm{C}}$ by doping different concentrations of PBA NPs, we concluded that the addition of 0.5 wt $\%$ of PBA NPs into the Felix $17 / 100$ material is suitable for achieving good electro-optical properties. 


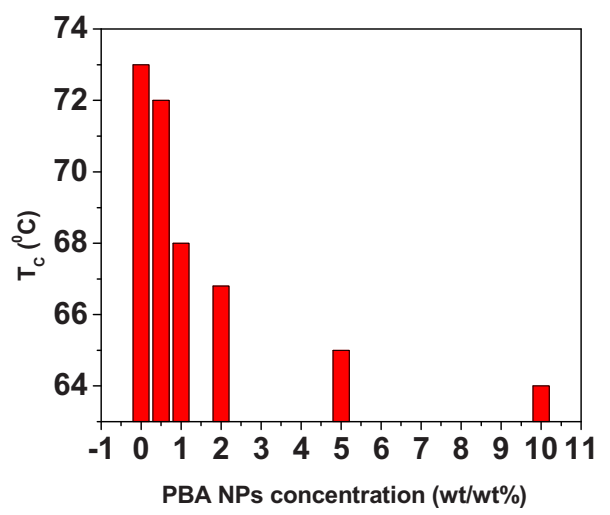

FIG. 10. (Color online) Change in $\mathrm{SmC}^{*}$-SmA $\mathrm{S}^{*}$ phase transition temperature $\left(T_{C}\right)$ of Felix 17/100 material doped with different concentrations of PBA NPs.

\section{CONCLUSIONS}

The PBA NPs induced HMT alignment of FLC molecules has been demonstrated experimentally. We observed that PBA NPs induced HMT alignment of FLC mixtures is the consequence of surface interaction between the ITO and PBA NPs in such a fashion that the PBA NPs nearly stand up on the ITO surface. The upright orientation of anthracene molecules provide template to FLC molecules in attaining a HMT alignment. We have also analyzed the effect of different PBA NPs concentrations on the physical parameters and dielectric relaxation processes of FLC Felix 17/100 in detail. We found that 0.5 wt $\%$ of PBA NPs dispersed in Felix 17/ 100 is suitable to achieve better electro-optical properties.

\section{ACKNOWLEDGMENTS}

The authors sincerely thank Professor R. C. Budhani, Director, National Physical Laboratory, for continuous encouragement and interest in this work. We sincerely thank Dr. S. S. Bawa, Dr. I. Coondoo, Dr. P. Goel, A. Choudhary, J. Prakash, A. Malik, T. Khan, and T. Joshi for fruitful discussions. The authors acknowledge to Dr. S. K. Dhawan of conducting polymer group for providing PBA NPs. The author (A.K.) is thankful to university grant commission (UGC) for providing financial assistance.
${ }^{1}$ X. Nie, R. Lu, H. Xianyu, T. X. Wu, and S.-S. Wu, J. Appl. Phys. 101, 103110 (2007).

${ }^{2}$ J. Cognard, Mol. Cryst. Liq. Cryst. Suppl. Ser. 1, 1 (1982).

${ }^{3}$ J. L. Janning, Appl. Phys. Lett. 21, 173 (1972).

${ }^{4}$ K. Hiroshima, Jpn. J. Appl. Phys., Part 2 21, L761 (1982).

${ }^{5}$ S.-C. Jeng, C.-W. Kuo, H.-L. Wang, and C.-C. Liao, Appl. Phys. Lett. 91, 061112 (2007).

${ }^{6}$ B. H. Hwang, H. J. Ahn, S. J. Rho, S. S. Chae, and H. K. Baik, Langmuir 25, 8306 (2009).

${ }^{7}$ H. J. Ahn, S. J. Rho, K. C. Kim, B. J. Kim, B. H. Hwang, C. J. Park, and H. K. Baik, Jpn. J. Appl. Phys., Part 1 44, 4092 (2005).

${ }^{8}$ C. Chen, J. E. Anderson, and P. J. Bos, Jpn. J. Appl. Phys., Part 2 44, L1126 (2005).

${ }^{9}$ Y. Shiraishi, N. Toshima, K. Maeda, H. Yoshikawa, J. Xu, and S. Kobayashi, Appl. Phys. Lett. 81, 2845 (2002).

${ }^{10}$ J. Prakash, A. Choudhary, A. Kumar, D. S. Mehta, and A. M. Biradar, Appl. Phys. Lett. 93, 112904 (2008).

${ }^{11}$ J. Prakash, A. Choudhary, D. S. Mehta, and A. M. Biradar, Phys. Rev. E 80, 012701 (2009).

${ }^{12}$ R. Dhar, A. S. Pandey, M. B. Pandey, S. Kumar, and R. Dabrowski, Appl. Phys. Express 1, 121501 (2008).

${ }^{13}$ W. Lee, C.-Y. Wang, and Y.-C. Shih, Appl. Phys. Lett. 85, 513 (2004).

${ }^{14}$ A. Kumar, J. Prakash, D. S. Mehta, W. Hasse, and A. M. Biradar, Appl. Phys. Lett. 85, 1 (2009).

${ }^{15}$ L.-S. Baik, S. Y. Jeon, S. H. Lee, K. A. Park, S. H. Jeong, K. H. An, and Y. H. Lee, Appl. Phys. Lett. 87, 263110 (2005).

${ }^{16}$ S.-J. Hwang, S.-C. Jeng, C.-Y. Yang, C.-W. Kuo, and C.-C. Liao, J. Phys. D: Appl. Phys. 42, 025102 (2009).

${ }^{17}$ A. Kumar, J. Prakash, P. Goel, T. Khan, S. K. Dhawan, P. Silotia, and A. M. Biradar, EPL 88, 26003 (2009).

${ }^{18}$ S. K. Kwon, J. Heo, J. W. Park, Y. H. Kim, H. Y. Oh, and S. T. Kim, SID Int. Symp. Digest Tech. Papers 36, 835 (2005).

${ }^{19}$ C. A. Landis, S. R. Parkin, and J. E. Anthony, Jpn. J. Appl. Phys., Part 1 44, 3921 (2005).

${ }^{20}$ H. Tang, Y. Li, X. Wang, and R. Sun, Semicond. Sci. Technol. 22, 287 (2007).

${ }^{21}$ G. Witte and C. Wöll, J. Mater. Res. 19, 1889 (2004).

${ }^{22}$ N. M. Buckanie and F. J. Mayer zu Heringdorf, Surf. Sci. 601, 1701 (2007).

${ }^{23}$ J. S. Jo, M. Ozaki, and K. Yoshino, Mol. Cryst. Liq. Cryst. 410, 191 (2004).

${ }^{24}$ G. Singh, G. V. Prakash, S. Kaur, A. Choudhary, and A. M. Biradar, Physica B 403, 3316 (2008).

${ }^{25}$ A. Dwivedi, R. Dhar, R. Dabrowski, and M. Tykarska, J. Phys. D: Appl. Phys. 42, 095402 (2009).

${ }^{26}$ G. Pandey, R. Dhar, V. K. Agrawal, and R. Dabrowski, Physica B 393, 167 (2007). 